\title{
MULTITEMPORAL CLASSIFICATION OF NATURAL VEGETATION COVER IN BRAZILIAN CERRADO
}

\author{
Gustavo Bayma Siqueira Silval, Marcio Pupin Mello ${ }^{2}$, Yosio Edemir Shimabukuro ${ }^{2}$, \\ Bernardo Friedrich Theodor Rudorff ${ }^{2}$, Daniel de Castro Victoria ${ }^{1}$ \\ ${ }^{1}$ Brazilian Agricultural Research Organization, EMBRAPA Satellite Monitoring \\ Avenida Soldado Passarinho, 303 - Campinas, SP, 13070-115, Brazil \\ bayma@cnpm.embrapa.br; daniel@cnpm.embrapa.br \\ ${ }^{2}$ National Institute for Space Research (INPE), Remote Sensing Division, \\ Avenida dos Astronautas, 1758 - São José dos Campos, SP, 12227-010, Brazil \\ mello@ieee.org; yosio@dsr.inpe.br; bernardo@dsr.inpe.br
}

\begin{abstract}
Spectral-Temporal Analysis by Response Surface (STARS), which utilizes surface response to represent time series spectral-temporal behavior of pixels in satellite images, was used to map and discriminate savanna vegetation classes in portion of Cerrado biome of Mato Grosso State, Brazil, using MODIS data. STARS utilized 16 daily MODIS, cloud-free, images that were collected from September 1st 2008 to August 31st 2009. The Multi-Coefficient Image (MCI) resulted from the STARS was used as input attributes for the three tested classifiers: (i) ML - maximum likelihood; (ii) SVM - support vector machine; and (iii) NN - neural network. The results showed that the NN classifier presented higher kappa coefficient (0.58) and overall accuracy of $68.6 \%$.
\end{abstract}

Index Terms - Savanna, Linear Spectral Mixture Model, STARS.

\section{INTRODUCTION}

Savannas are ecosystems characterized by the presence of a continuous layer of herbaceous vegetation with a discontinuous cover of shrubs and trees. The land use in savannas areas is diverse across the countries but predominate the collection and removal of trees, extensive livestock farming and cultivation of species of the annual cycle [1]. It is necessary to develop new tools to assist in vegetation mapping and monitoring knowledgment of large areas of vegetation cover, as Cerrado biome [2]. Precise and accurate measurements of seasonal dynamic of vegetation cover are fundamental to understanding the functioning of these ecosystems and to implement sustainable development practices. To analyze better the human disturbance dynamic in this biome, it is necessary to develop and adopt effective methods of evaluation and monitoring via change detection, to provide appropriate classification of land use and land cover [3].

Due to spectral dynamic the savanna natural classes Cerrado biome in Brazil could be mapped if the analysis takes account the dynamics over time. The SpectralTemporal Analysis by Response Surface (STARS) is a new multitemporal remote sensing classification method that utilizes surface response to represent time series spectraltemporal behaviour of pixels in satellite images. In this context, the main objective of this work is to apply the STARS to map and discriminate savanna vegetation classes in portion of Cerrado biome of Mato Grosso State, Brazil, using MODIS data.

\section{SPECTRAL-TEMPORAL ANALYSIS BY RESPONSE SURFACE}

The Spectral-Temporal Analysis by Response Surface (STARS) is a method to represent the full information content of multitemporal-multispectral remote sensing images in a single synthetic image (MCI: Multi-Coefficient Image) that can be used for classification purposes. Assuming that each pixel has a spectral profile in a single image, an image time-series will has $T$ spectral profiles over time for each pixel, where $T$ is the number of images at $T$ different dates. Thus, at each pixel we have the spectraltemporal information that can be modeled as a multiple linear regression (response surface) with two independent variables: time $(t)$ and spectrum $(s)$; and one dependent variable representing the observed values of the sensor (r), such as reflectance or band transformation (e.g. mixing model fraction images). One model used by Vieira [4] to fit the response surface is the Polynomial Trend Surface (PTS), which generates a smoother surface representing gradual changes. The system of simultaneous linear equations for the PTS model using the degree $d$ has the form 


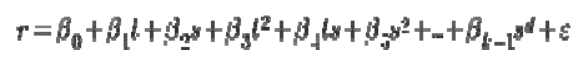

where the $k$ coefficients are denoted by $\beta_{\mathrm{i}}$; and the $\varepsilon$ term represents the error vector assumed to be uncorrelated with mean equal zero and variance equal to the residual mean square. The number of coefficients is given in function of $d$ as

$$
h=\frac{(d-1)(d+2)}{2}
$$

These $k$ fitted coefficient at each pixel will compose the Multi-Coefficient Image (MCI). These coefficients represent the information content of the spectral-temporal response surface of a pixel. For example, the $\beta_{0}$ represents the surface offset with regard to the origin of the $r$ variable [5]. An overview of STARS is presented in Fig. 1.

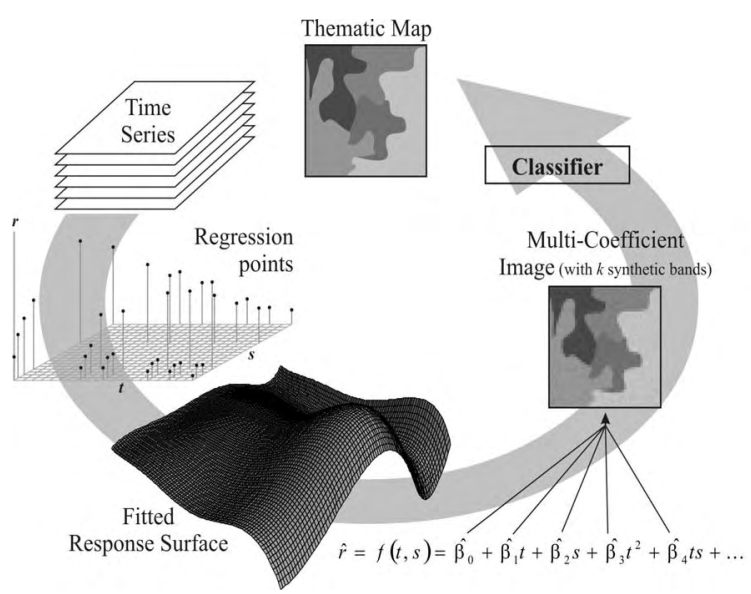

Fig. 1. Flowchart of the adopted methodology.

\section{DESCRIPTION OF THE STUDY SITE}

The study area corresponds to an area of $31,250 \mathrm{~km}^{2}$ in Mato Grosso State, comprising all the main Cerrado formations: forestlands, shrublands and grasslands (Fig. 2). Cerrado biome occupies an area of approximately 206 million hectares, located in the central portion of Brazil.

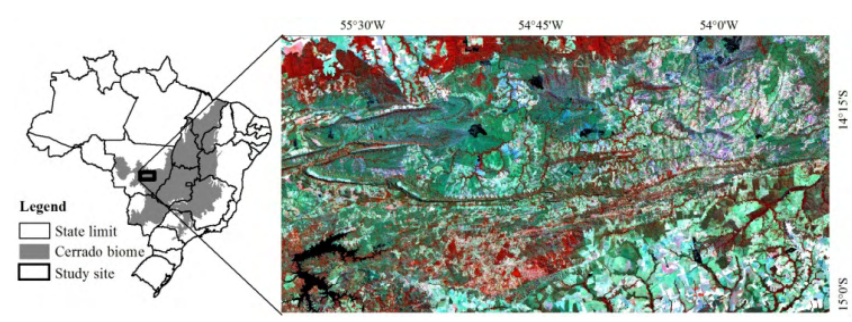

Fig. 2. Location (SAD69) of the study area, color composite 4(R), 5 (G), 3 (B) TM/Landsat-5, acquired in July 2008.

\section{MATERIALS AND METHODS}

To construct the reference map was used the Cerrado biome vegetation map that was elaborated thorough the interpretation of 2001 and 2002 Landsat ETM+ satellite images [6]. The vegetation map was updated till 2009, where the new deforested areas were delimited. The vegetation classes selected are: Cerrado Woodland (CW); Wooded Cerrado (WC); Shrub Cerrado (SC); and Cerrado grassland (CG). Daily MODIS, cloud-free, images were collected from September 1st 2008 to August 31st 2009. From 365 possible images, 16 cloud-free images were selected. After the daily MODIS images selection was applied the Linear Spectral Mixing Model (LSMM) [7] to generate vegetation, soil and shade fraction images. These fraction images time series were then used as input data to STARS.

The variables for STARS were: $(t)$ the image date acquisition, given in Julian day; $(s)$ arbitrary labelled as 0 , 0.5 and 1 for soil, vegetation and shade association, respectively; and $(r)$ the proportion value estimated by the LSMM, varying from 0 to 1 . In order to standardize all variables to the same magnitude, the variable $t$ was rescaled to a closed interval between 0 and 1, as suggested by Vieira [4]. The STARS was then performed using the PTS model with $d=5$ using the orthonormal coefficients [8]. With $d=5$, the PTS model has 21 coefficients $(k=21)$ that will compose, pixel by pixel, the 21 synthetic bands of the MCI, which were used as input attributes for classifiers.

Three classifiers already implemented in the ENVI software version 4.5 [9] were used: (i) ML - Maximum Likelihood; (ii) SVM - Support Vector Machine; and (iii) NN - Neural Network. A reference image was used to randomly select the training samples (1000 pixels per class) and the accuracy assessment samples (160 per class, calculated by the multinomial distribution [10]). The 160 samples per class were collected independently and used to compute the confusion matrix, which enabled compute the kappa $(\kappa)$ estimation and their variances $\left(\sigma_{\kappa}\right)$. Statistical tests, based on the standardized Gaussian distribution ( $Z$ distribution) were performed for testing both the individually significance of each kappa index and the differences (pairwise tests) among the classifiers performance, as presented by Congalton \& Green [10].

\section{RESULTS AND DISCUSSION}

The classification results are presented in Table 1. SVM and $\mathrm{NN}$ classifier presented the higher kappa coefficients $(0.58$ and 0.55 , respectively) followed by ML (0.37). A Z-test was performed to assess the classifiers' performance. Values greater than 1.96 on the diagonal indicate that the classification was better than a random classification at twosided and 5\% significance level. Values higher than 1.96 off 
the diagonal indicated significant difference between each evaluated pair of classifiers. $Z$ tests indicated then that results of SVM and NN were similar and better than ML.

To evaluate the performance of using MCI, which is the result of the STARS as input for the classifiers, one Neural Network classification was performed directly (without STARS) in the 48 LSMM fraction (3 fraction x 16 dates). The kappa value of 0.20 , probably as a consequence of the Hughes phenomenon [11], indicates that STARS succeed in synthesizing the full information content of entire time series in a single synthetic image, which reduced the data dimensionality from 48 LSMM fraction images to 21 synthetic bands in the MCI.

Table 1. Results of the $Z$ tests comparing classifiers' performance.

\begin{tabular}{cccc} 
& ML & SVM & NN \\
$\kappa$ & 0.3688 & 0.5521 & 0.5813 \\
$\sigma_{\kappa} \times 10^{4}$ & 5.6410 & 5.6410 & 5.9076 \\
$\mathbf{M L}$ & 15.53 & & \\
$\mathbf{S V M}$ & 5.33 & 22.22 & \\
$\mathbf{N N}$ & 6.35 & 0.88 & 23.91 \\
& & \multicolumn{3}{c}{ Grey cell indicate significance at $5 \%}$.
\end{tabular}

The confusion matrixes related to the three classifications (ML, SVM and NN classifiers) are presented in Tables 2, 3 and 4 . Table 2 refers to the ML and showed that vegetation classes of CW and CG obtained better results than WC and SC. This results points that in vegetation classes that predominated only trees $(\mathrm{CW})$ or grass $(\mathrm{CG})$ the classifier worked well, $68.75 \%$ and $91.25 \%$ of accuracy, respectively. SVM confusion matrix (Table 3) showed similar tendency to classify with the highest accuracy $\mathrm{CW}(75.63 \%)$ and $\mathrm{CG}$ $(73.75 \%)$ classes. WC and SC that are vegetation classes that predominates a mixture of trees and shrubs had better results in SMV than in ML classification In NN classifier the WC class showed better accuracy (66.25\%) than ML and SVM classifiers, although SC demonstrated lower accuracy $(48.75 \%)$ than SVM classifier.

$\mathrm{NN}$ and SVM classifiers presented the higher overall accuracy ( $68.6 \%$ and $66.4 \%$, respectively) followed by ML $(52.7 \%)$. The $\mathrm{NN}$ tends to produce better results than the ML classifier because it does not require Gaussian statistical data distribution. The neural network adapts well to any distribution, while the ML assumes that the data have normal distribution. Training samples of $\mathrm{NN}$ affect more than ML classifier, i.e., if there is one outlier in the training sample that leads to greater loss of performance in NN than in ML. The results indicated that the classes that have lower species diversity in their composition performed better than the classes that were more mixed.
Table 2. ML classifier confusion matrix.

\begin{tabular}{cccccc}
\hline ML classifier & CW & WC & SC & CG & Total \\
\hline CW & $\mathbf{1 1 0}$ & 25 & 9 & 6 & $\mathbf{1 5 0}$ \\
WC & 24 & $\mathbf{4 8}$ & 13 & 6 & $\mathbf{9 1}$ \\
SG & 8 & 13 & $\mathbf{3 3}$ & 2 & $\mathbf{5 6}$ \\
CG & 18 & 74 & 105 & $\mathbf{1 4 6}$ & $\mathbf{3 4 3}$ \\
\hline Total & $\mathbf{1 6 0}$ & $\mathbf{1 6 0}$ & $\mathbf{1 6 0}$ & $\mathbf{1 6 0}$ & $\mathbf{6 4 0}$ \\
\hline \multicolumn{5}{c}{ Overall Accuracy $=52.7 \%$}
\end{tabular}

Table 3. SVM classifier confusion matrix.

\begin{tabular}{cccccc}
\hline SVM classifier & CW & WC & SC & CG & Total \\
\hline CW & $\mathbf{1 2 1}$ & 28 & 14 & 9 & $\mathbf{1 7 2}$ \\
WC & 17 & $\mathbf{9 0}$ & 29 & 13 & $\mathbf{1 4 9}$ \\
SG & 12 & 31 & $\mathbf{9 6}$ & 20 & $\mathbf{1 5 9}$ \\
CG & 10 & 11 & 21 & $\mathbf{1 1 8}$ & $\mathbf{1 6 0}$ \\
\hline Total & $\mathbf{1 6 0}$ & $\mathbf{1 6 0}$ & $\mathbf{1 6 0}$ & $\mathbf{1 6 0}$ & $\mathbf{6 4 0}$ \\
\hline \multicolumn{5}{c}{ Overall Accuracy $=66.4 \%$}
\end{tabular}

Table 4. NN classifier confusion matrix.

\begin{tabular}{cccccc}
\hline NN classifier & CW & WC & SC & CG & Total \\
\hline CW & $\mathbf{1 2 3}$ & 30 & 8 & 9 & $\mathbf{1 7 0}$ \\
WC & 18 & $\mathbf{1 0 6}$ & 48 & 4 & $\mathbf{1 7 6}$ \\
SG & 12 & 19 & $\mathbf{7 8}$ & 15 & $\mathbf{1 2 4}$ \\
CG & 7 & 5 & 26 & $\mathbf{1 3 2}$ & $\mathbf{1 7 0}$ \\
\hline Total & $\mathbf{1 6 0}$ & $\mathbf{1 6 0}$ & $\mathbf{1 6 0}$ & $\mathbf{1 6 0}$ & $\mathbf{6 4 0}$ \\
\hline \multicolumn{5}{c}{ Overall Accuracy $=68.6 \%$}
\end{tabular}

\section{CONCLUSION}

The proposed methodology has shown promising in discriminating and mapping the Cerrado savanna classes. STARS MCI synthetic bands classified by NN presented better results than ML and SVM classifiers. The results showed that STARS was able to discriminate Cerrado savanna classes due to the capability to represent these classes through fitted spectral-temporal response surfaces. In the future we plan to investigate the classifications tuning the classifiers in order to better select their parameters. 


\section{REFERENCES}

[1] W.J. Goedert; E. Wagner; A. O. Barcellos. Savanas tropicais: dimensão, histórico e perspectivas. In: F.G. Faleiro; A.L. Farias Neto. Savanas: desafios e estratégias para o equilíbrio entre sociedade, agronegócio e recursos naturais. Planaltina: Embrapa Cerrados, Cap. 1, pp. 49-77, 2008.

[2] A.M. Nepomuceno; A.N.C. S. Rosa; P.R. Meneses. Reconhecimento de Fitofisionomias do Cerrado no Parque Nacional de Brasília através de Classificação de Imagens de Radar de Abertura Sintética. In: Simpósio Brasileiro de Sensoriamento Remoto, 11. (SBSR), 2003, Belo Horizonte, MG Anais... São José dos Campos: INPE pp. 2823-2830, 2003.

[3] W. Jepson. "A disappearing biome? Reconsidering land-cover change in the Brazilian savanna", The Geographical Journal, v. 171, n. 2, pp. 99-111, 2005.

[4] C.A.O. Vieira. "Accuracy of remotely sensing classification of agricultural crops: a comparative study", Ph.D. Thesis, University of Nottingham, Nottingham, UK, 2000.

[5] M.H. Kutner; C.J. Nachtsheim; J. Neter; W. Li. Applied linear statistical models, 5th ed. New York, USA: McGraw-Hill, 1992.

[6] E.E. Sano; R. Rosa; J.L. Brito; L.G. Ferreira Jr, "Land cover mapping of the tropical savanna region in Brazil", Environmental Monitoring and Assessment, v. 166, p. 113-124, 2009.

[7] Y.E. Shimabukuro; J.A. Smith. "The least-squares mixing models to generate fraction images derived from remote sensing multispectral data", IEEE Transactions on Geoscience and Remote Sensing, v.29, n.1, pp. 16-20, 1991.

[8] P.M. Mather, Computational methods of multivariate analysis in physical geography, John Wiley \& Sons, Chichester, UK, 1976.

[9] RSI. Environment for Visualizing Images - ENVI. ver. 4.5. Boulder, CO, USA: ITT Industries. 2008.

[10] R.G. Congalton and K. Green, Assessing the accuracy of remotely sensed data: principles and pratices, 2nd ed. Boca Raton, FL, USA: CRC Press, 2009.

[11] G. Hughes, "On the mean accuracy of statistical pattern recognizers," IEEE Trans. Inf. Theory, vol. 14, no. 1, pp. 55-63, Jan. 1968. 